\title{
RSBHCWT: Re-Sampling Binary Hex Code Windowing Technique to Enhance Target Detection
}

\author{
R. K. Singh ${ }^{1 *}$, D. Elizabath Rani ${ }^{2}$ and Syed Jalal Ahmad ${ }^{1}$ \\ 'GNITC, Hyderabadad - 501506, Telangana, India; rk@gniindia.org, Jalal0000@yahoo.com \\ ²GITAM University, Visakhapatnam - 530045, Andhra Pradesh, India; hod_ei@gitam.edu
}

\begin{abstract}
Objectives: To Design Doppler tolerant radar codes to enhance multiple moving targets. Methods/Statistical Analysis: In this paper we present a novel mathematical approach to improve the target detection in Doppler tolerant radar. The presented approach in this paper is called Re-Sampling Binary Hex Code Windowing Technique (RSBHCWT). This approach not only increases the amplitude of main lobe, but also decreases the amplitude of side lobs to create multiple windows at desired Doppler. We use mat lab to validate our results through simulation. Findings: We developed a windowing approach that can detect multiple moving targets. Application/Improvements: The presented model is useful in military applications. Our approach is on top of oversampled PTM code by creating number of windows with respect to desired Doppler's.
\end{abstract}

Keywords: Hex Codes, Mat Lab, Re Sampling, Windows

\section{Introduction}

Radar system uses the transmission of the electromagnetic signal and receives the return echoes received from the target, to determine the range and velocity of the target in terms of delay and frequency shift from the transmitted pulses. The received echoed signals are processed using match filtering technique with the transmitted signal at different desired delay to generate a setback Doppler record. The ultimate outcome of match filtering technique is frequently described as a thumbtack shape, with maximum amplitude at the exact delay depicting the range of the target from the radar but introduce unwanted side lobes at various delays and Doppler. Efforts have been made to reduce these side lobes using techniques like barker codes, minimum peak side lobes codes etc. By the use of certain special coding techniques one can minimize the effect of side lobes at certain areas of Doppler plan up to certain limit. However this may not always true, as the received signal echo is weak and also has Doppler shift, target may be masked if it will occur within the range side lobe of a huge returns. Golay proposed an approach called Golay complementary sequences in which the author proved that the sum of the auto correlation of Golay complementary sequences vanishes at every non-zero digit delays. This indicates that the sum of the uncertainty functions (composite uncertainty function) of the Golay code sequences is free from lobs along with the zero Doppler line. This does not permit unambiguous range imaging. This methodology makes the sequences ideal for radar range imaging for static targets. However in a real world practices, a major setback exists to adopt such type of complementary sequences in radar system. As the ideal auto correlation property of such sequences are very much sensitive to Doppler shift. At the non -zero Doppler sifts; the combined uncertainty function of complementary sequences can have large effect of side lobs masking actual targets in various delays. This doesn't allow unambiguous range imaging. All most all such sequences include several complimentary sequences and poly-phase sequences undergo same problem of higher side lobes at Doppler frequency shits for practical

${ }^{*}$ Author for correspondence 
targets. Some of the common examples of poly-phase sequences that show some acceptance to Doppler are frank sequences/p1, p2, p3 and p4 sequences/and $\mathrm{p}(\mathrm{n}, \mathrm{k})$ sequences/. Also some of the sub complementary codes is another type, which is very near to complementary codes. Such type of code sequences has some tolerance against Doppler. However they are not simple to be generated. No doubt PTM sequence get window at zero Doppler, however the size of the window is small, difficult to detect the target at various Doppler's caused due to practical moving targets, and consume more power, as resampling starts from single bit. Therefore cannot be used effectively.

So in this paper a novel technique is being presented to improve the target detection probability for multiple moving targets using windowing approach. This technique is simple but very much effective to enhance the SNR and power of the received echoes, by the use of Re- Sampling Binary Hex Code windowing Technique (RBHCWT). In this approach we minimize the effect of noise at desired Doppler and increase the size of the window at the desired Doppler. This approach can be very much effective to detect the position of the moving targets at various ranges at effective Doppler for vide range of delays. Proposed an approach called Complementary Code Matrix (CCM), in which the authors construct a matrix having the ability to make the aperiodic autocorrelation side lobes to zero. However this approach does not perform well for moving targets. In ${ }^{\beta-9}$ the authors presented a code based on "pushing sequences" to generate wave forms, in which almost all ambiguity functions are free from side lobes. Such approaches works on frequency hopping at very rapid velocity, so in general these approaches cannot eliminate side lobes for all ranges within a Doppler interval. 10 Proposed a method for constructing radar waveforms by the use of PAM and OFDAM techniques, which are free from side lobs. However the complexity of the algorithm increased in case of multiple targets, which increases delay and is not suitable for fast moving targets. Also use PTM, oversampled PTMs and Golay sampled PTM codes to create windows at Doppler frequencies. However these Doppler windows were very small for which range is clear for all delays. Phase coded Pulse waveforms seems to be very high-quality substitutes for radar waveforms, as they present high time-bandwidth product. Such coding techniques have stable modulus and can be simply developed and used for radar waveforms. Several such radar waveforms have already been presented earlier ${ }^{11-14}$ with high-quality autocorrelation and cross correlation properties. Such waveforms are particularly considered for getting excellent delay and Doppler response characteristics. However care has not been taken to get spatial resolution characteristics. But this drawback can be overcome with the help of proper scheming of the phase coded waveforms by optimizing the MIMO uncertainty function, which improves the delay, Doppler and also take care to get the unique property of the resolution

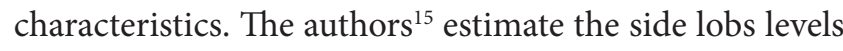
as a criterion to use the radar in noisy environment. In this approach the authors use wide band binary wave forms for transmission; however this approach did not reduce the effect of side lobs, which results in poor response to detect the moving targets. The writer modifies the match filtering technique to reduce the effect of side lobesto. This approach enhances the quality of service of radar if the targets are stationary. But if the targets are moving this approach increases the complexity, which increases delay. In proposed a performance evaluation for target detection in Blackout conditions. However this model works well only when the target is stationary, so cannot have optimal use for moving target detection. The proposed phased MIMO radar for improvement in target detection by exploiting coherence signal processing gain and waveform diversity However this particular approach fails to find multiple moving targets.

The rest of the paper is organized as follows. In section 2 proposed approaches has been discussed in detail. In section 3 we present the simulation results and conclusion of paper is discussed in section 4 .

\section{Proposed Approach}

In this approach we use the equal weighted binary hex codes (four digit binary codes) up to decimal value of 15 (i.e. 0 -15) for simplicity and to reduce power by combining 4 bits (Hex Code) together and then resample the code. Also equal weighted hex will reduce the D.C. component as well as make it compatible over physical channel. So the total such codes will be six from 0 to 16 . This approach is simple, but very much effective to detect the multiple moving targets. Our approach increases the amplitude of main lobe and also decreases the amplitudes of the side lobes to increase the SNR of radar signal and resulted in creating number of Doppler windows. 
Initially we consider an input sequence of equal weighted binary hex codes of decimal numbers from $0-15$ which are divisible by three. So the sequence can be represented as

$\prod_{i=\mathbf{1}}^{N} X i$ Where $\mathrm{X}=3$

\section{And $\mathrm{i}=1$ to $\mathrm{N} 1 \leq \mathrm{N} \leq 4$}

The remaining missing decimal numbers of equal weight from $0-15$ can be calculated from the equation 2

$\mathrm{Y}=\frac{\mathbf{I}_{\mathrm{T}}+\mathbf{L}_{\mathrm{T}}}{\mathrm{I}_{\mathrm{T}}}$

Where $\mathrm{I}_{\mathrm{T}}=$ first term of the above series i.e. 3 and $\mathrm{L}_{\mathrm{T}}$ is the last decimal numbers of the series calculated from equation 1 i.e. 12

And the next decimal number can be calculated as

$\mathrm{C}=2 \mathrm{Y}$

We increase all equal weighted hex codes up to the length of 256 bits to get very good signal to noise ratio by using our approach (i.e. RBHCWT). There sampling was done by appending the complementary code at every stage with the main code using Complementary Coding Technique (CCT). The code can be further increased for optimization for particular resolution and signal to noise ration.

Mathematically the length of the code word can be calculated as

$\mathrm{L}=(\mathrm{P})^{4}$

Where $\mathrm{P}=$ hex code length (i.e. 4)

Above result revels that the length of the starting code word is 4 bits and can be represented as

$\mathrm{S}_{\mathrm{CW}}=\mathrm{H}_{\mathrm{C} 1}$

$\mathrm{S}_{\mathrm{CW}}=$ Starting Code

Let $\mathrm{H}_{\mathrm{C} 1}=0011$ Then

$\mathrm{S}_{\mathrm{C} 2}=\mathrm{H}_{\mathrm{C} 1} \overline{H_{C_{1}}}$

The next code word can be represented as $\mathrm{S}_{\mathrm{C} 3}=\mathrm{S}_{\mathrm{C} 2} \overline{S_{\mathrm{C} \mathbf{2}}}$

This process continues till the length of the code word reaches the final value (i.e. 256 bits) or
$\mathrm{L}=(\mathrm{P})^{4}$ by the use of CCT and we get the final code word and can be represented as

$\mathrm{S}_{\mathrm{Cn}}=\mathrm{S}_{\mathrm{C}(\mathrm{n}-1)} \overline{\mathbf{S}_{\mathbf{c}(\mathrm{n}-\Xi)}}$

By the use of equations 5, 6, 7 and 8 we get the final code word who's starting hex code (i.e. $\mathrm{H}_{\mathrm{Cl}}$ ) is 0011 . Similarly other codes for reaming set of hex code series can be generated. All the 6 hex codes in the final code formation (i.e. up to 256 bits length) are tested for target detection using mat lab simulation tool.

\section{Simulation Results}

We analyze the results with the help of mat lab. Figure 1 to Figure 6 shows the variation of Delay with respect to Doppler, whose starting hex codes are 0011, 0101, 0110, 1001, 1010, and 1100. The length of all codes increases up to 256 bits with the help of BRSHCWT (see, Esq. 5, 6, 7 , and 8). Use of these codes have resulted in very large windows where the noise is almost $90 \mathrm{db}$ down and the targets can be easily detected for the entire delay covering the complete range of the radar. The detection at specified Doppler and any minor variation of the Doppler does not affect the detection process which is almost free from range side lobes. For major portion of practical Doppler the detection and continuous tracking is possible without hindrance. Also after main window we are getting number of windows one after another and only on some spot frequencies the side lobe is above $0.2 \mathrm{db}$.

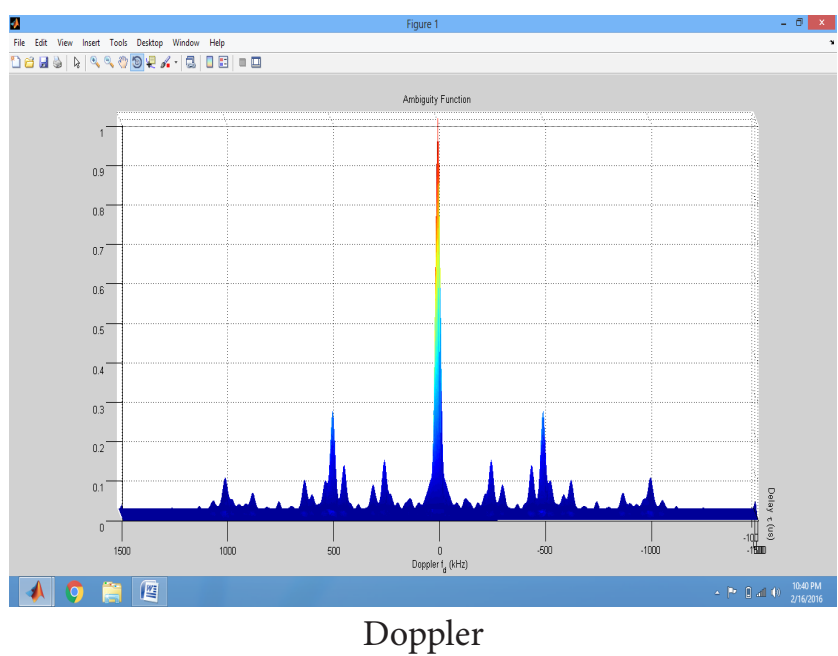

Figure 1. Delay vs. Doppler (Starting code 0011). 


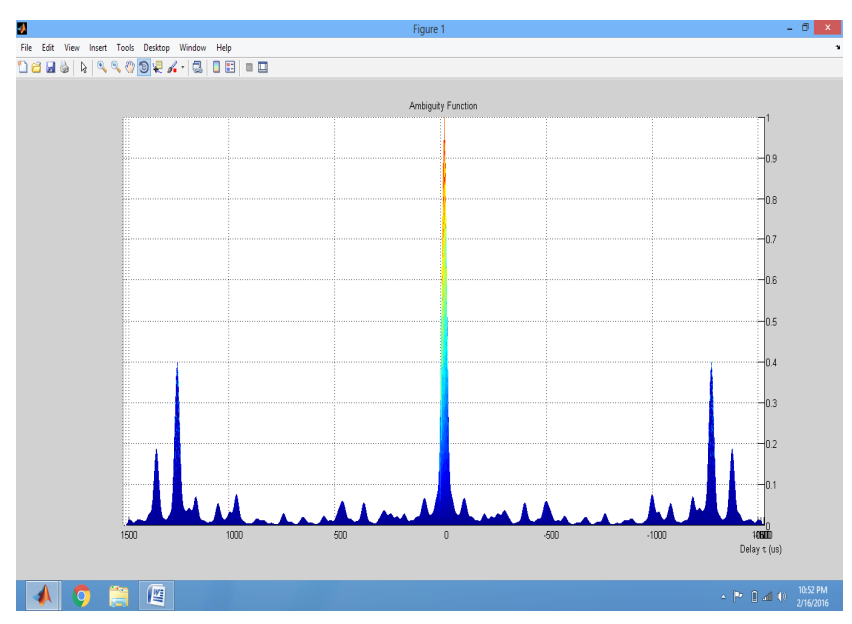

Doppler

Figure 2. Delay vs. Doppler (Starting code 0101).

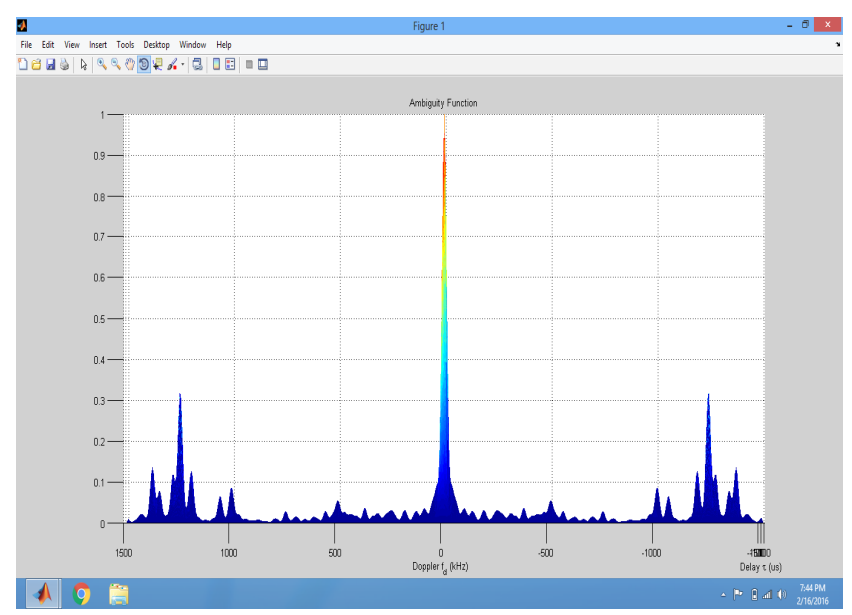

Doppler

Figure 3. Delay vs. Doppler (Starting code 0110).

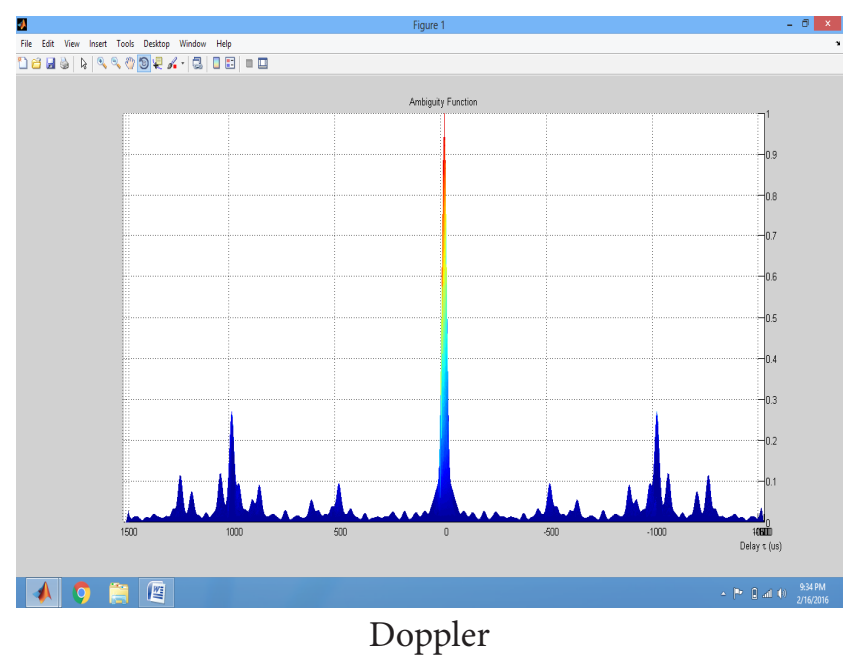

Figure 4. Delay vs. Doppler (Starting code 1001).

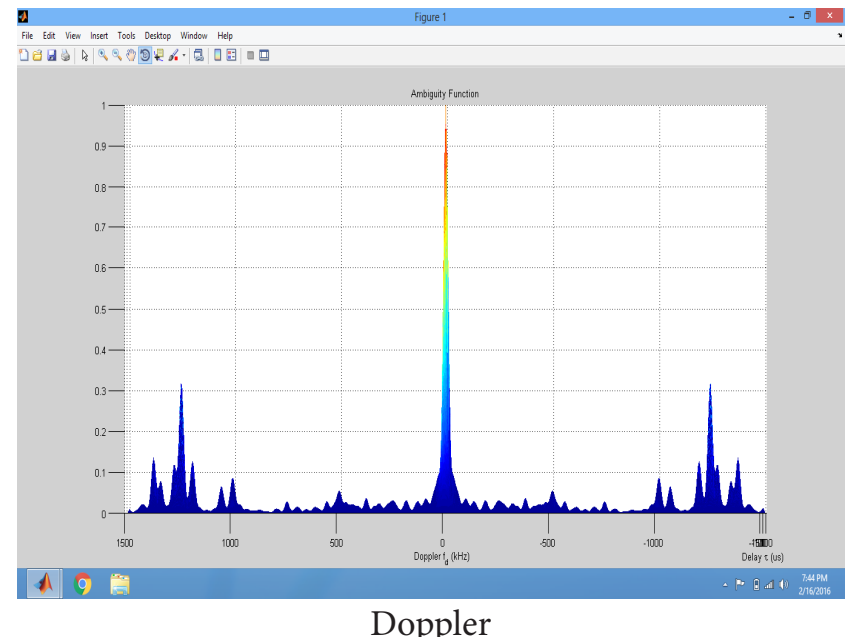

Figure 5. Delay vs. Doppler (Starting code 1010).

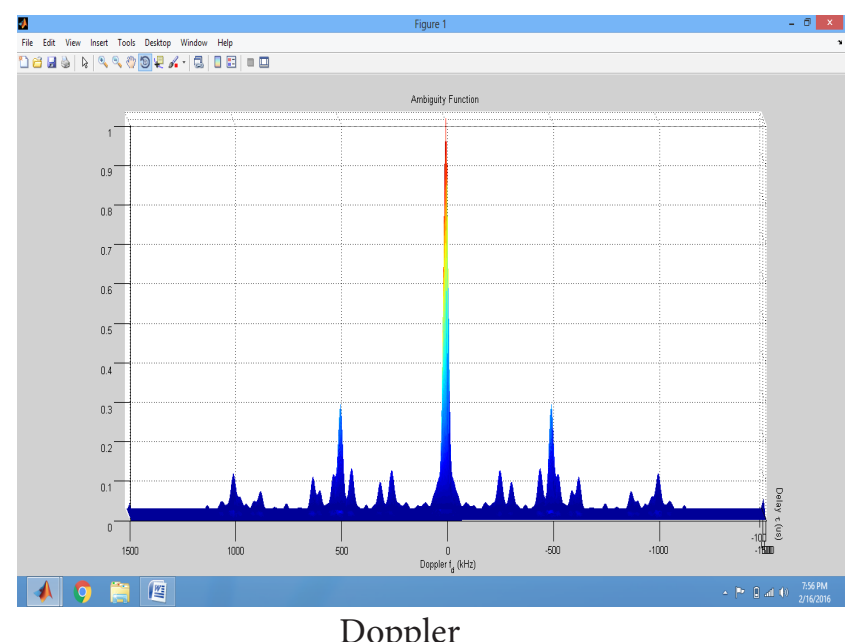

Figure 6. Delay vs. Doppler (Starting code 1100).

From all six figures we observe that if two consecutive values of either zeroes or ones at the starting or ending of starting hex code of the binary re sampled hex coding technique the variation of delay versus Doppler will be little bit effected by the side lobs. The side lobes are prominent at certain spot Doppler frequencies in the complete range of Doppler shifts. The results are best with 0101 and 1010 where for large windows of Doppler frequencies the side lobes are below $90 \mathrm{db}$ down giving very good detection for the entire range of the radar. For the codes having two similar bits at the middle of starting hex code of RBHCWT the results are good for the general Doppler frequencies under consideration for practical targets and have clear windows for the complete range of radar. 


\section{Conclusion}

In radar transmission, improved Quality of Service can be obtained by minimizing the effect of the side lobs, so that the size of the window should be increased over a large extent to get a desired Doppler. In this paper, we presented a mathematical model to compute the sequence of weighted binary hex codes. We also presented simulation results, which demonstrate that with the use of weighted binary hex codes the percentage of side lobs decreases and on the same time percentage of getting huge window size is also increases thereby, reduction in the noise. This paper also increases the peak of the main lobe, which increases the signal strength to detect the moving target with a minimal delay.

\section{References}

1. Golay MJE. Complementary series. IRE Trans Inform Theory. $1961 ; 7(2): 82-7.2$.

2. Frank RL. Polyphase codes with good non-periodic correlation properties. IEEE Trans. Inform Theory. 1963; 9(1):43-5.

3. Kretschmer FF, Lewis BL. Doppler properties of poly-phase coded pulse-compression waveforms. IEEE Transition Aerospace Electronic System. 19831; 19(4):521 -31.

4. Felhauer T. Design and analysis of new $\mathrm{P}(\mathrm{n}, \mathrm{k})$ poly-phase pulse compression codes. IEEE Trans Aerosp Electron Syst. 1994; 30(3):865-74.

5. Sivaswami R. Self-clutter cancellation and ambiguity properties of sub complementary sequences. IEEE Trans Aerospace Electron Syst. 1982; 18(2):163-81.

6. Guey JH, Bell MR. Diversity waveform sets for delay-Doppler imaging. IEEE Trans Information Theory. 1998; 44(4):1504 -22.
7. Coxson GE. Construction of complementary code matrices for waveform design. IEEE Transaction on Aerospace and Electrical Systems. 2013 Jul; 49(3):1806-16.

8. Chang C, Bell MR. Frequency-coded waveforms for enhanced delay-Doppler resolution. IEEE Trans on Information Theory. 2003; 49(11):2960-71.

9. Costas JP. A study of a class of detection waveforms having nearly ideal range-Doppler ambiguity properties. Proceedings of IEEE; 1984. p. 996-1009.

10. Pezeshki A, Calderbank R, Scharf LL. Side lobe suppression in a desired range/Doppler interval. IEEE Radar Conference; 2009. p. 1-5.

11. Deng H. Polyphase code design for orthogonal netted radar systems. IEEE Trans Signal Process. 2004; 52(11):3126-35.

12. Deng H. Synthesis of binary sequences with good autocorrelation and cross-correlation properties by simulated annealing. IEEE Trans Aerospace Electronic System. 1996; 32:98-107.

13. Sharma GVK, Raja Rajeswari K. Four phase orthogonal code design for MIMO radar systems. IEEE National Conference on Communications, NCC; 2012.

14. Liu B, He Z, Zeng J, Liu B. Polyphase orthogonal code design for MIMO radar systems. Proceedings of CIE International Conference Radar; 2006 Oct.

15. Alejos AV, Dawood M, Mohammed HU. Estimation of side lobe level variations of phased codes in presence of random interference for Bi static wideband noise radar. International Journal of Antennas and Propagation. 2015:11.

16. Levanon. Creating side lobe-free range zone around detected radar target. Electrical and Electronics Engineers in Israel (IEEEI); 2014. p. 1-5.

17. Li Y, Li X, Wang HQ, Deng B, Qin Y. Performance evaluation of target detection with a near-space vehicle-borne radar in blackout condition. Sensors Open Access Journal. 2016; 16(1):1-13.

18. Sur SN, Bera R, Maji B. Phased-MIMO radar for improvement in target detection. Indian Journal of Science and Technology. 2016 Oct; 9(40):1-4. 